\title{
Modified Spherical Wave Functions With Anisotropy Ratio: Application to the Analysis of Scattering by Multilayered Anisotropic Shells
}

\author{
Cheng-Wei Qiu, Saïd Zouhdi, Senior Member, IEEE, and Adel Razek, Fellow, IEEE
}

\begin{abstract}
We describe a novel and rigorous vector eigenfunction expansion of electric-type Green's dyadics for radially multilayered uniaxial anisotropic media in terms of the modified spherical vector wave functions, which can take into account the effects of anisotropy ratio systematically. In each layer, the material constitutions $\bar{\epsilon}$ and $\bar{\mu}$ are tensors and distribution of sources is arbitrary. Both the unbounded and scattering dyadic Green's functions (DGFs) for rotationally uniaxial anisotropic media are derived in spherical coordinates $(r, \theta, \phi)$. The coefficients of scattering DGFs, based on the coupling recursive algorithm satisfied by the coefficient matrix, are derived and expressed in a compact form. With these DGFs obtained, the electromagnetic fields in each layer are straightforward once the current source is known. A specific model is proposed for the scattering and absorption characteristics of multilayered uniaxial anisotropic spheres, and some novel performance regarding anisotropy effects is revealed.
\end{abstract}

Index Terms-Anisotropic ratio, dyadic Green's functions (DGFs), modified spherical wave functions, radially multilayered structures, recurrence matrix, scattering and absorption, vector eigenfunction expansion.

\section{INTRODUCTION}

$\mathbf{T}$ HE dyadic Green's functions (DGFs) technique [1]-[3] has been widely used to characterize electromagnetic wave propagation and to solve electromagnetic boundary value problems for the last decades. The dyadic Green's function serves as a kernel of the integral and has to be defined or formulated beforehand. However, with the complexity of media growing, the dyadic Green's function representations for media also become more complicated. In recent years, due to the advances in material science and technology which have manifested fabrication of various kinds of complex materials, considerable attention has been paid to the interaction of electromagnetic waves with anisotropic materials [4]-[6], bianisotropic media [7] and chirowaveguides [8].

In addition to the modal representation of DGFs in [9] and [10], vector eigenfunction expansion of DGFs was established

Manuscript received June 28, 2007; revised August 22, 2007.

C. W. Qiu is with the Laboratoire de Génie Electrique de Paris, SUPELEC, 91192 Gif-Sur-Yvette Cedex, France and also with the Department of Electrical and Computer Engineering, National University of Singapore, NUS, Singapore 117576, Singapore.

S. Zouhdi is with the University Paris Sud, Orsay 91405, Cedex, France and also with the Laboratoire de Génie Electrique de Paris, SUPELEC, Gif-SurYvette, Cedex, France (e-mail: said.zouhdi@supelec.fr).

A. Razek is with the Laboratoire de Génie Electrique de Paris, SUPELEC, 91192 Gif-Sur-Yvette Cedex, France.

Digital Object Identifier 10.1109/TAP.2007.910491 for isotropic media [2]. For planarly multilayered media, DGFs have been derived [11]-[13]. For cylindrical multilayered media, DGFs were constructed for chiral media [14]. However, due to the complexity of the parameter tensors, plane wave expansion along with the Fourier transform and the theory of TE and TM decomposition are widely employed in the analysis of anisotropic media [15]. For the same reason, when formulating the DGFs in anisotropic media, most papers express Green's dyadics in Cartesian or cylindrical coordinates such as [16], [17]. Due to the complexity of DGF formulation for multilayered structures in spherical coordinates, only some related pieces of work have been done for isotropic [18], [19] and bi-isotropic media [20]. Conventionally, only the case of single layered anisotropic sphere with plane-wave incidence can be studied [21], [22], and the application could be limited unless the excitation can be a current source or dipole. Even if the method of eigenfunction expansion is tailored for multilayered anisotropic spheres [23], the formulation becomes rather lengthy if one wants to take into account anisotropy in Mie theory. Besides, if both permittivity and permeability possess anisotropy, as in the present paper, the methodology adopted in [23] would be quite cumbersome to apply. Moreover, the role of anisotropy in the scattering properties deserves further investigation, so we propose the parameter of anisotropic ratios to characterize such effects. In our approach, not only the scattering due to arbitrary current distribution and multilayered anisotropic spheres is computed, but also the scattering problem of plane-wave incidence can be transformed into a radiation problem by introducing a special dipole so as to employ obtained DGFs thereafter. Hence, the conventional plane-wave scattering in the presence of an anisotropic sphere can be treated as only a special subset of our work. Furthermore, the sphere's anisotropy ratio effects on radar cross section are taken into account in a compact form by modifying the spherical wave functions, which avoids tedious mathematical formulation.

This paper aims at solving radiation and scattering from an embedded source of excitation in an arbitrary layer of the radially multilayered anisotropic shells. Starting from potential formulation, we obtain the field representations and unbounded DGFs in terms of modified spherical wave functions. The spectral-domain EM DGFs are derived by considering multiple transmission and reflection at each interface. A specific numerical example is provided with the particular interest in anisotropy effects, and the effects of anisotropy ratio are shown. The originality, compactness and generality of the proposed theory are the main contributions of the current work. 


\section{BASic Formulations OF Potentials AND Modified SPHERICAL WAVE FUNCTIONS}

In this work, we investigate a kind of general uniaxial media which consists of constitutive tensors of permittivity and permeability in the form

$$
\begin{aligned}
\overline{\boldsymbol{\epsilon}} & =\epsilon_{0}\left[\left(\epsilon_{r}-\epsilon_{t}\right) \hat{\boldsymbol{r}} \hat{\boldsymbol{r}}+\epsilon_{t} \overline{\boldsymbol{I}}\right] \\
\overline{\boldsymbol{\mu}} & =\mu_{0}\left[\left(\mu_{r}-\mu_{t}\right) \hat{\boldsymbol{r}} \hat{\boldsymbol{r}}+\mu_{t} \overline{\boldsymbol{I}}\right]
\end{aligned}
$$

where the unit vector dyad is $\overline{\boldsymbol{I}}=\hat{\boldsymbol{r}} \hat{\boldsymbol{r}}+\hat{\boldsymbol{\theta}} \hat{\boldsymbol{\theta}}+\hat{\boldsymbol{\phi}} \hat{\boldsymbol{\phi}}$. To our knowledge, this kind of form was first introduced in [24] where $\hat{\boldsymbol{\theta}} \hat{\boldsymbol{\phi}}$ and $\hat{\boldsymbol{\phi}} \hat{\boldsymbol{\theta}}$ components are not zero. This kind of anisotropy can be either natural or introduced in the processing of the surface plane and the shear. We notice that if the nondiagonal components of the material tensors $\overline{\boldsymbol{\epsilon}}$ and $\overline{\boldsymbol{\mu}}$ are zero, then the rotations would be equivalent to letting $\hat{\boldsymbol{r}} \hat{\boldsymbol{r}}$ unchanged while rotating the transverse elements (to $\hat{\boldsymbol{r}}$ ) with $\hat{\boldsymbol{r}}$ as axes. The material in our study remains invariant under such a rotation, which was called G-type [24] where the analysis was in 2-D with respect to $\hat{z}$ as the axis of rotation. Thus, for this uniaxial anisotropic material, the anisotropy ratio (AR) can be defined as

$$
\begin{aligned}
\mathrm{AR}_{e} & =\epsilon_{t} / \epsilon_{r} \\
\mathrm{AR}_{m} & =\mu_{t} / \mu_{r}
\end{aligned}
$$

where the subscripts $e$ and $m$ denote electric and magnetic anisotropy ratios, respectively. For anisotropic media, the Maxwell equations and the constitutive relationship are given as follows:

$$
\begin{aligned}
\nabla \times \boldsymbol{E} & =-i \omega \overline{\boldsymbol{\mu}} \cdot \boldsymbol{H} \\
\nabla \times \boldsymbol{H} & =i \omega \overline{\boldsymbol{\epsilon}} \cdot \boldsymbol{E}+\boldsymbol{J}
\end{aligned}
$$

where time dependence is $e^{i \omega t}$ and is suppressed.

In the source-free case, (3) can be rewritten as

$$
\begin{aligned}
\nabla \times\left(\overline{\boldsymbol{\epsilon}}^{-1} \cdot \boldsymbol{D}\right) & =-i \omega \boldsymbol{B} \\
\nabla \times\left(\overline{\boldsymbol{\mu}}^{-1} \cdot \boldsymbol{B}\right) & =i \omega \boldsymbol{D} .
\end{aligned}
$$

From (4), we have the idea to express $\boldsymbol{B}$ and $\boldsymbol{D}$ in terms of the following sets:

$$
\begin{aligned}
\boldsymbol{B}_{\mathrm{TM}} & =\nabla \times\left(\hat{\boldsymbol{r}} \psi_{\mathrm{TM}}\right) \\
\boldsymbol{D}_{\mathrm{TE}} & =-\nabla \times\left(\hat{\boldsymbol{r}} \psi_{\mathrm{TE}}\right)
\end{aligned}
$$

and the TM and TE modes are with respect to $\hat{r}$ in the spherical coordinate.

Substituting (5) into (4), we obtain

$$
\begin{aligned}
\boldsymbol{B}_{\mathrm{TE}} & =\frac{1}{i \omega}\left[\nabla \times\left(\overline{\boldsymbol{\epsilon}}^{-1} \cdot \nabla \times\left(\hat{\boldsymbol{r}} \psi_{\mathrm{TE}}\right)\right)\right] \\
\boldsymbol{D}_{\mathrm{TM}} & =\frac{1}{i \omega}\left[\nabla \times\left(\overline{\boldsymbol{\mu}}^{-1} \cdot \nabla \times\left(\hat{\boldsymbol{r}} \psi_{\mathrm{TM}}\right)\right)\right] .
\end{aligned}
$$

By inserting (6a) and (5b) into (4b) and equating the radial components, the potential $\psi_{\mathrm{TE}}$ can be obtained. The potential $\psi_{\mathrm{TM}}$ can be obtained in a similar way by substituting (6b) and (5a) into (4a)

$$
\begin{aligned}
& \frac{1}{\mathrm{AR}_{e}} \frac{\partial^{2} \psi_{\mathrm{TM}}}{\partial r^{2}}+\nabla_{t}^{2} \psi_{\mathrm{TM}}+\omega^{2} \mu_{0} \epsilon_{0} \mu_{t} \epsilon_{r} \psi_{\mathrm{TM}}=0 \\
& \frac{1}{\mathrm{AR}_{m}} \frac{\partial^{2} \psi_{\mathrm{TE}}}{\partial r^{2}}+\nabla_{t}^{2} \psi_{\mathrm{TE}}+\omega^{2} \mu_{0} \epsilon_{0} \mu_{r} \epsilon_{t} \psi_{\mathrm{TE}}=0
\end{aligned}
$$

where

$$
\nabla_{t}^{2}=\frac{1}{r^{2} \sin \theta} \frac{\partial}{\partial \theta}\left(\sin \theta \frac{\partial}{\partial \theta}\right)+\frac{1}{r^{2} \sin ^{2} \theta} \frac{\partial^{2}}{\partial \phi^{2}} .
$$

Using the separation of variables method, we find that the solutions to the above equations are composed of superpositions of Bessel functions, associated Legendre polynomials, and harmonic functions, i.e.,

$$
\begin{aligned}
\psi_{\mathrm{TM}} & =\sum_{m, n} a_{m, n} j_{v_{1}}\left(k_{t} r\right) P_{n}^{m}(\cos \theta){ }_{\sin }^{\cos } m \phi \\
\psi_{\mathrm{TE}} & =\sum_{m, n} b_{m, n} j_{v_{2}}\left(k_{t} r\right) P_{n}^{m}(\cos \theta){ }_{\sin }^{\cos } m \phi \\
v_{1} & =\left[n(n+1) \frac{\epsilon_{t}}{\epsilon_{r}}+\frac{1}{4}\right]^{1 / 2}-\frac{1}{2} \\
v_{2} & =\left[n(n+1) \frac{\mu_{t}}{\mu_{r}}+\frac{1}{4}\right]^{1 / 2}-\frac{1}{2} \\
k_{t} & =\omega \sqrt{\epsilon_{0} \mu_{0} \epsilon_{t} \mu_{t}}
\end{aligned}
$$

where $j_{v}(\cdot)$ is spherical Bessel functions. The field representations can be obtained by using TE/TM decomposition

$$
\begin{aligned}
\boldsymbol{E}^{\mathrm{TE}}= & \frac{-1}{\epsilon_{0} \epsilon_{t} r \sin \theta} \frac{\partial \psi_{\mathrm{TE}}}{\partial \phi} \hat{\boldsymbol{\theta}}+\frac{1}{\epsilon_{0} \epsilon_{t} r} \frac{\partial \psi_{\mathrm{TE}}}{\partial \theta} \hat{\boldsymbol{\phi}} \\
\boldsymbol{H}^{\mathrm{TE}}= & \frac{\omega}{i k_{t}^{2}}\left(\frac{\partial^{2}}{\partial r^{2}}+k_{t}^{2}\right) \psi_{\mathrm{TE}} \hat{\boldsymbol{r}}+\frac{\omega}{i k_{t}^{2} r} \frac{\partial^{2} \psi_{\mathrm{TE}} \hat{\boldsymbol{\theta}}}{\partial r \partial \theta} \\
& +\frac{\omega}{i k_{t}^{2} r \sin \theta} \frac{\partial^{2} \psi_{\mathrm{TE}}}{\partial r \partial \phi} \hat{\boldsymbol{\phi}} \\
\boldsymbol{E}^{\mathrm{TM}}= & \frac{\omega}{i k_{t}^{2}}\left(\frac{\partial^{2}}{\partial r^{2}}+k_{t}^{2}\right) \psi_{\mathrm{TM}} \hat{\boldsymbol{r}}+\frac{\omega}{i k_{t}^{2} r} \frac{\partial^{2} \psi_{\mathrm{TM}}}{\partial r \partial \theta} \hat{\boldsymbol{\theta}} \\
& +\frac{\omega}{i k_{t}^{2} r \sin \theta} \frac{\partial^{2} \psi_{\mathrm{TM}}}{\partial r \partial \phi} \hat{\boldsymbol{\phi}} \\
\boldsymbol{H}^{\mathrm{TM}}= & \frac{1}{\mu_{0} \mu_{t} r \sin \theta} \frac{\partial \psi_{\mathrm{TM}}}{\partial \phi} \hat{\boldsymbol{\theta}} \\
& -\frac{1}{\mu_{0} \mu_{t} r} \frac{\partial \psi_{\mathrm{TM}}}{\partial \theta} \hat{\boldsymbol{\phi}} .
\end{aligned}
$$

After some manipulation, we obtain

$$
\begin{aligned}
\boldsymbol{E}= & \boldsymbol{E}^{\mathrm{TE}}+\boldsymbol{E}^{\mathrm{TM}} \\
= & \sum_{n, m} D_{m n}\left[b_{e_{o} m n} \boldsymbol{M}_{e_{o} m v_{2}}^{(l)}\left(k_{t}\right)\right. \\
& \left.+a_{e_{o} m n} \boldsymbol{N}_{e_{o} m v_{1}}^{(l)}\left(k_{t}\right)\right] \\
\boldsymbol{H}= & \boldsymbol{H}^{\mathrm{TE}}+\boldsymbol{H}^{\mathrm{TM}} \\
= & \frac{-i}{\eta_{t}} \sum_{n, m} D_{m n}\left[a_{o} m n \boldsymbol{M}_{o}^{(l)} v_{v_{1}}\left(k_{t}\right)\right. \\
& \left.+b_{o} m n \boldsymbol{N}_{e_{o} m v_{2}}^{(l)}\left(k_{t}\right)\right] \\
D_{m n}= & \frac{\left(2-\delta_{m}^{0}\right)(2 n+1)(n-m) !}{4 n(n+1)(n+m) !}
\end{aligned}
$$


where the superscript $l$ denotes the kinds of Bessel/Hankel functions, $\eta_{t}=\sqrt{\mu_{t} / \epsilon_{t}}$, and

$$
\begin{aligned}
\boldsymbol{M}_{o m v}^{(l)}(k)= & \mp \frac{m z_{v}^{(l)}(k r)}{\sin \theta} P_{n}^{m}(\cos \theta) \frac{\sin }{\cos } m \phi \hat{\boldsymbol{\theta}} \\
& -z_{v}^{(l)}(k r) \frac{d P_{n}^{m}(\cos \theta)}{d \theta} \operatorname{sis}_{\sin }^{\cos } m \phi \hat{\boldsymbol{\phi}} \\
\boldsymbol{N}_{e m v}^{(l)}(k)= & \frac{v(v+1) z_{v}^{(l)}(k r)}{k r} P_{n}^{m}(\cos \theta){ }_{\sin }^{\cos } m \phi \hat{\boldsymbol{r}} \\
& +\frac{\partial\left[r z_{v}^{(l)}(k r)\right]}{k r \partial r}\left[\frac{d P_{n}^{m}(\cos \theta)}{d \theta} \sin _{\sin }^{\cos } m \phi \hat{\boldsymbol{\theta}}\right. \\
& \left.\mp \frac{m}{\sin \theta} P_{n}^{m}(\cos \theta) \frac{\sin }{\cos } m \phi \hat{\boldsymbol{\phi}}\right] .
\end{aligned}
$$

In our paper, $z_{v}^{(l)}(x)$ are defined as

$$
z_{v}^{(l)}(x)=\left\{\begin{array}{ll}
j_{v}(x), & l=1 \\
h_{v}^{(2)}(x), & l=2
\end{array} .\right.
$$

\section{GENERAL EXPRESSION OF DYADIC GREEN'S FUNCTIONS}

The modified vector wave functions in (12) for rotationally symmetric anisotropic media can also be used as vector eigenfunctions to expand and express the DGFs in unbounded or multilayered cases. Without the loss of generality, both $\overline{\boldsymbol{\epsilon}}$ and $\bar{\mu}$ have the uniaxial form, which results in

$$
\begin{aligned}
& v_{1}\left(v_{1}+1\right)=n(n+1) \mathrm{AR}_{e} \\
& v_{2}\left(v_{2}+1\right)=n(n+1) \mathrm{AR}_{m} .
\end{aligned}
$$

The electric field can be expressed in terms of electric-type DGF and current source

$$
\boldsymbol{E}=-i \omega \int_{V^{\prime}} \overline{\boldsymbol{G}}_{E J} \cdot \boldsymbol{J}\left(\boldsymbol{r}^{\prime}\right) d V^{\prime}
$$

where $V^{\prime}$ represents the source volume. The source distribution of $\boldsymbol{J}(\boldsymbol{r})$ in (3) can also be expressed as

$$
\boldsymbol{J}(\boldsymbol{r})=\int_{V^{\prime}} \overline{\mathbf{I}} \delta\left(\boldsymbol{r}-\boldsymbol{r}^{\prime}\right) \cdot \boldsymbol{J}\left(\boldsymbol{r}^{\prime}\right) d V^{\prime}
$$

Inserting (15) and (16) into (3), we have

$$
\nabla \times\left[\overline{\boldsymbol{\mu}}^{-1} \cdot \nabla \times \overline{\boldsymbol{G}}_{E J}\right]-\omega^{2} \overline{\boldsymbol{\epsilon}} \cdot \overline{\boldsymbol{G}}_{E J}=\overline{\boldsymbol{I}} \delta\left(\boldsymbol{r}-\boldsymbol{r}^{\prime}\right) .
$$

By means of the vector eigenfunction expansion, one can finally arrive at

$$
\begin{aligned}
\overline{\boldsymbol{G}}_{E J}=-\frac{1}{\omega^{2} \epsilon_{0} \epsilon_{r}} \hat{\boldsymbol{r}} \hat{\boldsymbol{r}} \delta\left(r-r^{\prime}\right)+\frac{i \mu_{0} \mu_{t} k_{t}}{4 \pi} \sum_{m, n} D_{m n} \\
\quad \times\left\{\begin{array}{l}
\boldsymbol{M}_{e_{o} m v_{2}}^{(2)}\left(k_{t}\right) \boldsymbol{M}_{e_{m} m v_{2}}^{\prime}\left(k_{t}\right)+\boldsymbol{N}_{e_{m} m v_{1}}^{(2)}\left(k_{t}\right) \boldsymbol{N}_{e_{m} m v_{1}}^{\prime}\left(k_{t}\right) \\
\boldsymbol{M}_{e_{o} m v_{2}}\left(k_{t}\right) \boldsymbol{M}_{e_{o} m v_{2}}^{\prime(2)}\left(k_{t}\right)+\boldsymbol{N}_{e_{o} m v_{1}}\left(k_{t}\right) \boldsymbol{N}_{e_{o} m v_{1}}^{\prime(2)}\left(k_{t}\right)
\end{array}\right.
\end{aligned}
$$

where the upper part is for $r>r^{\prime}$ and the lower part is for $r<r^{\prime}$, and $\boldsymbol{M}_{e m v}^{(2)}$ denotes the second kind of Hankel function used herewith and $\boldsymbol{M}_{e_{m} m v}$ represents the first kind of Bessel function involved. Note that the irrotational part has been extracted. It is obvious that (18) is reducible to the isotropic case $\left(\epsilon_{r}=\epsilon_{t}\right.$ and $\left.\mu_{r}=\mu_{t}\right)$ [3] and the present dyadic Green's function agrees with the reduced form. Using the method of scattering superposition, the dyadic Green's function can be considered as the sum of the unbounded and scattering DGFs. The former corresponds to the contribution due to the source in the infinite homogeneous space while the latter reflects the contribution of the source due to the presence of multiple interfaces. The DGFs is thus given as

$$
\overline{\boldsymbol{G}}_{e}^{(f s)}\left(\boldsymbol{r}, \boldsymbol{r}^{\prime}\right)=\overline{\boldsymbol{G}}_{0}\left(\boldsymbol{r}, \boldsymbol{r}^{\prime}\right) \delta_{f}^{s}+\overline{\boldsymbol{G}}_{s}^{(f s)}\left(\boldsymbol{r}, \boldsymbol{r}^{\prime}\right)
$$

where $\overline{\boldsymbol{G}}_{e}$ and $\overline{\boldsymbol{G}}_{0}$ denote the total and unbounded electric DGFs, respectively; superscripts $f$ and $s$ denote the field point located at $f$ th layer and source located at $s$ th layer, respectively; and $\delta_{f}^{s}$ is the Kronecker delta function. Consider a radially N-layered geometry of a uniaxial anisotropic shell shown in Fig. 1. The permittivity, permeability and wave number in $f$ th layer are defined as

$$
\begin{aligned}
\overline{\boldsymbol{\epsilon}}_{f} & =\epsilon_{0}\left[\left(\epsilon_{r, f}-\epsilon_{t, f}\right) \hat{\boldsymbol{r}} \hat{\boldsymbol{r}}+\epsilon_{t, f} \bar{I}\right] \\
\overline{\boldsymbol{\mu}}_{f} & =\mu_{0}\left[\left(\mu_{r, f}-\mu_{t, f}\right) \hat{\boldsymbol{r}} \hat{\boldsymbol{r}}+\mu_{t, f} \overline{\boldsymbol{I}}\right] \\
k_{t, f}^{2} & =\omega^{2} \epsilon_{0} \mu_{0} \epsilon_{t, f} \mu_{t, f} .
\end{aligned}
$$

Assuming that the current source is located in sth layer, we may construct the scattering DGFs as follows by considering the model of multiple transmission and reflection due to the interfaces

$$
\begin{aligned}
& \overline{\boldsymbol{G}}_{e s}^{(f s)}\left(\boldsymbol{r}, \boldsymbol{r}^{\prime}\right) \\
& =\frac{i \mu_{0} \mu_{t, f} k_{t, s}}{4 \pi} \sum_{m, n} D_{m n}\left\{\left(1-\delta_{f}^{N}\right) \boldsymbol{M}_{e_{o} m v_{2, f}}^{(2)}\left(k_{t, f}\right)\right. \\
& \times\left[\left(1-\delta_{s}^{1}\right) A_{M}^{f s} \boldsymbol{M}_{e_{o}}^{\prime} m v_{2, s}\left(k_{t, s}\right)\right. \\
& \left.+\left(1-\delta_{s}^{N}\right) B_{M}^{f s} \boldsymbol{M}_{o}^{\prime(2)} v_{2, s}\left(k_{t, s}\right)\right] \\
& +\left(1-\delta_{f}^{N}\right) \boldsymbol{N}_{e}^{(2)} m v_{1, f}\left(k_{t, f}\right) \\
& \times\left[\left(1-\delta_{s}^{1}\right) A_{N}^{f s} \boldsymbol{N}_{e}^{\prime} m v_{1, s}\left(k_{t, s}\right)\right. \\
& \left.+\left(1-\delta_{s}^{N}\right) B_{N}^{f s} \boldsymbol{N}_{o}^{\prime(2)} v_{1, s}\left(k_{t, s}\right)\right] \\
& +\left(1-\delta_{f}^{1}\right) \boldsymbol{M}_{o} m v_{2, f}\left(k_{t, f}\right) \\
& \times\left[\left(1-\delta_{s}^{1}\right) C_{M}^{f s} \boldsymbol{M}_{e}^{\prime} m v_{2, s}\left(k_{t, s}\right)\right. \\
& \left.+\left(1-\delta_{s}^{N}\right) D_{M}^{f s} \boldsymbol{M}_{o}^{\prime(2)} m v_{2, s}\left(k_{t, s}\right)\right] \\
& +\left(1-\delta_{f}^{1}\right) \boldsymbol{N}_{o} m v_{1, f}\left(k_{t, f}\right) \\
& \times\left[\left(1-\delta_{s}^{1}\right) C_{N}^{f s} \boldsymbol{N}_{e_{o}}^{\prime} m v_{1 s}\left(k_{t, s}\right)\right. \\
& \left.\left.+\left(1-\delta_{s}^{N}\right) D_{N}^{f s} \boldsymbol{N}_{o}^{\prime(2)} m v_{1, s}\left(k_{t, s}\right)\right]\right\}
\end{aligned}
$$

where $A_{M, N}^{f s}, B_{M, N}^{f s}, C_{M, N}^{f s}$ and $D_{M, N}^{f s}$ are the coefficients of scattered DGFs to be determined by the boundary conditions at each interface. The physical insight of the above equation 


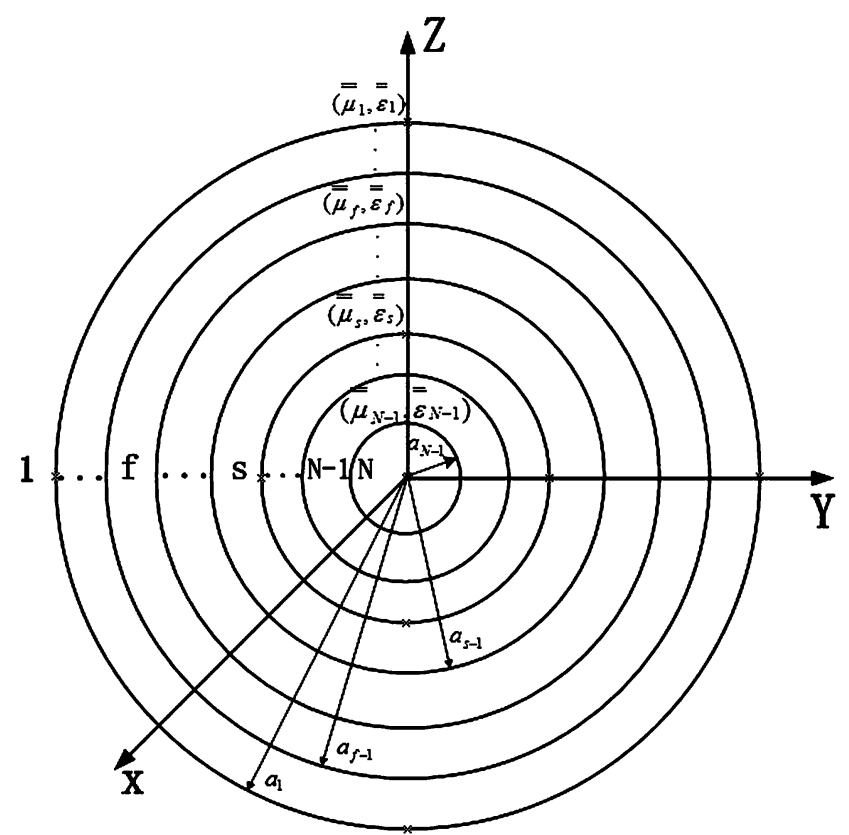

Fig. 1. Geometry of radially multilayered media.

resides in the fact that in the $f$ th layer the scattered fields are composed of inward and outward waves, which are due to the reflections from the outer interfaces at $a_{1}, \ldots, a_{f-1}$ and inner interfaces at $a_{f}, \ldots, a_{N-1}$, respectively.

\section{Determination OF SCATtering DGFs' COEFFicients: A RECURSIVE MATRIX METHOD}

\section{A. Recursive Algorithms}

By the proposed recursive matrix method, all the unknown scattering coefficients can be determined by applying boundary conditions at $r=a_{f}(\mathrm{f}=1,2, \ldots, \mathrm{N}-1)$

$$
\begin{aligned}
\hat{\boldsymbol{r}} \times \overline{\boldsymbol{G}}_{e}^{(f+1) s} & =\hat{\boldsymbol{r}} \times \overline{\boldsymbol{G}}_{e}^{f s} \\
\hat{\boldsymbol{r}} \times\left[\overline{\boldsymbol{\mu}}_{f+1}^{-1} \cdot \nabla \times \overline{\boldsymbol{G}}_{e}^{(f+1) s}\right] & =\hat{\boldsymbol{r}} \times\left[\overline{\boldsymbol{\mu}}_{f}^{-1} \cdot \nabla \times \overline{\boldsymbol{G}}_{e}^{f s}\right] .
\end{aligned}
$$

To simplify the symbolic calculations, let us introduce the following operators:

$$
\begin{aligned}
\hbar_{q, i l} & =h_{v_{q, i}}^{(2)}\left(k_{t, i} a_{l}\right) \\
\Im_{q, i l} & =j_{v_{q, i}}\left(k_{t, i} a_{l}\right) \\
\partial \hbar_{q, i l} & =\left.\frac{d\left[x h_{v_{q, i}}^{(2)}(x)\right]}{x d x}\right|_{x=k_{t, i} a_{l}} \\
\partial \Im_{q, i l} & =\left.\frac{d\left[x j_{v_{q, i}}(x)\right]}{x d x}\right|_{x=k_{t, i} a_{l}}
\end{aligned}
$$

where $q=1,2$ indicate $v_{1}$ and $v_{2}$, respectively.

A set of linear equations of the scattering coefficients, which can be represented by a series of compact matrices, are constructed to demonstrate the boundary conditions clearly

$$
\begin{aligned}
{\left[F_{l,(f+1)}\right] \cdot\left\{\left[\Upsilon_{l,(f+1) s}\right]\right.} & \left.+\delta_{f+1}^{s}\left[U_{(f+1)}\right]\right\} \\
& =\left[F_{l, f}\right] \cdot\left\{\left[\Upsilon_{l, f s}\right]+\delta_{f}^{s}\left[D_{f}\right]\right\}
\end{aligned}
$$

where $l=M, N$ and

$$
\begin{aligned}
{\left[F_{M, f}\right] } & =\left[\begin{array}{cc}
\hbar_{2, f f} & \Im_{2, f f} \\
\frac{k_{t, f}}{\mu_{t, f}} \partial \hbar_{2, f f} & \frac{k_{t, f}}{\mu_{t, f}} \partial \Im_{2, f f}
\end{array}\right] \\
{\left[F_{N, f}\right] } & =\left[\begin{array}{cc}
\partial \hbar_{1, f f} & \partial \Im_{1, f f} \\
\frac{k_{t, f}}{\mu_{t, f}} \hbar_{1, f f} & \frac{k_{t, f}}{\mu_{t, f}} \Im_{1, f f}
\end{array}\right] \\
{\left[\Upsilon_{l f s}\right] } & =\left[\begin{array}{ll}
A_{l}^{f s} & B_{l}^{f s} \\
C_{l}^{f s} & D_{l}^{f s}
\end{array}\right] \\
{\left[U_{f}\right] } & =\left[\begin{array}{ll}
1 & 0 \\
0 & 0
\end{array}\right] \\
{\left[D_{f}\right] } & =\left[\begin{array}{ll}
0 & 0 \\
0 & 1
\end{array}\right] .
\end{aligned}
$$

Defining the following transmission T-matrix:

$$
\left[T_{l, f}\right]=\left[F_{l,(f+1)}\right]^{-1} \cdot\left[F_{l, f}\right]
$$

where $\left[F_{l,(f+1)}\right]^{-1}$ is the inverse matrix of $\left[F_{l,(f+1)}\right]$. We rewrite the linear equation into the following form:

$$
\begin{aligned}
{\left[\Upsilon_{l,(f+1) s}\right]=\left[T_{l, f}\right] \cdot\left\{\left[\Upsilon_{l f s}\right]+\delta_{f}^{s}\left[D_{f}\right]\right\} } & \\
& -\delta_{f+1}^{s}\left[U_{(f+1)}\right] .
\end{aligned}
$$

To shorten the expression, we also introduce

$$
\begin{aligned}
{\left[T_{l}^{K}\right]_{2 \times 2} } & =\left[T_{l, N-1}\right]\left[T_{l, N-2}\right] \cdots\left[T_{l, K+1}\right]\left[T_{l, K}\right] \\
& =\left[\begin{array}{ll}
T_{l, 11}^{K} & T_{l, 12}^{K} \\
T_{l, 21}^{K} & T_{l, 22}^{K}
\end{array}\right] .
\end{aligned}
$$

It should be noted that the coefficients matrices of the first and the last layers have the following relations:

$$
\begin{aligned}
{\left[\Upsilon_{l, 1 s}\right] } & =\left[\begin{array}{cc}
A_{l}^{1 s} & B_{l}^{1 s} \\
0 & 0
\end{array}\right] \\
{\left[\Upsilon_{l, N s}\right] } & =\left[\begin{array}{cc}
0 & 0 \\
C_{l}^{N s} & D_{l}^{N s}
\end{array}\right] .
\end{aligned}
$$

\section{B. Application to Specific Cases}

To illustrate how to apply the recursive algorithms of transmission and reflections coefficient matrices, the following cases are specifically considered where the source is located in the first, intermediate, and the last layers, respectively.

1) Source in the First Layer: When the current source is located in the first layer (i.e., $s=1)$, the terms containing $\left(1-\delta_{s}^{1}\right)$ in (21) vanishes. The coefficient matrices in (25c) and (29) will be further reduced to

$$
\begin{aligned}
& {\left[\Upsilon_{l, 11}\right]=\left[\begin{array}{cc}
0 & B_{l}^{11} \\
0 & 0
\end{array}\right]} \\
& {\left[\Upsilon_{l, f 1}\right]=\left[\begin{array}{cc}
0 & B_{l}^{f 1} \\
0 & D_{l}^{f 1}
\end{array}\right]} \\
& {\left[\Upsilon_{l, N 1}\right]=\left[\begin{array}{cc}
0 & 0 \\
0 & D_{l}^{N 1}
\end{array}\right]}
\end{aligned}
$$

where $f=2,3, \ldots, N-1$. It can be seen that only two coefficients for the first layer and the last layer, but four coefficients 
for each of the remaining layers, need to be solved for. By following (27), the recurrence relations in the $f$ th layer become

$$
\left[\Upsilon_{l, f 1}\right]=\left[T_{l, f-1}\right] \cdots\left[T_{l, 1}\right]\left\{\left[\Upsilon_{l, 11}\right]+\left[D_{1}\right]\right\} .
$$

With $f=N$ in (31), a matrix equation satisfied by the coefficient matrices in (30) can be obtained. The coefficients for the first layer is given by

$$
B_{l}^{11}=-\frac{T_{l, 12}^{(1)}}{T_{l, 11}^{(1)}} .
$$

The coefficients for the last layer can be derived in terms of the coefficients for the first layer given by

$$
D_{l}^{N 1}=T_{l, 21}^{(1)} B_{l}^{11}+T_{l, 22}^{(1)} .
$$

The coefficients for the intermediate layers can be then obtained by substituting the coefficients for the first layer in (32) to (31). Thus, all the coefficients can be obtained by these procedures.

2) Source in the Intermediate Layers: When the current source is located in an intermediate layer, (i.e., $s \neq 1, N$ ), only the terms containing $\left(1-\delta_{f}^{1}\right)$ for the first layer or $\left(1-\delta_{f}^{N}\right)$ for the last layer vanish in (21). The coefficient matrices in $(25 \mathrm{c})$ and (29) will be further reduced to

$$
\begin{aligned}
{\left[\Upsilon_{l, 1 s}\right] } & =\left[\begin{array}{cc}
A_{l}^{1 s} & B_{l}^{1 s} \\
0 & 0
\end{array}\right] \\
{\left[\Upsilon_{l, f s}\right] } & =\left[\begin{array}{ll}
A_{l}^{f s} & B_{l}^{f s} \\
C_{l}^{f s} & D_{l}^{f s}
\end{array}\right] \\
{\left[\Upsilon_{l, N s}\right] } & =\left[\begin{array}{cc}
0 & 0 \\
C_{l}^{N s} & D_{l}^{N s}
\end{array}\right] .
\end{aligned}
$$

From (27), the recurrence equation becomes

$$
\begin{array}{r}
{\left[\Upsilon_{l, f s}\right]=\left[T_{l, f-1}\right] \cdots\left[T_{l, s}\right] \cdot\left\{\left[T_{l, s-1}\right] \cdots\left[T_{l, 1}\right]\left[\Upsilon_{l, 1 s}\right]\right.} \\
\left.+u(f-s-1)\left[D_{s}\right]-u(f-s)\left[U_{s}\right]\right\}
\end{array}
$$

where $u\left(x-x_{0}\right)$ is the unit step function. For $f=N$, the coefficients for the first layer are given by

$$
\begin{gathered}
A_{l}^{1 s}=\frac{T_{l, 11}^{(s)}}{T_{l, 11}^{(1)}} \\
B_{l}^{1 s}=-\frac{T_{l, 12}^{(s)}}{T_{l, 11}^{(1)}}
\end{gathered}
$$

and those for the last layer are

$$
\begin{aligned}
& C_{l}^{N s}=T_{l, 21}^{(1)} A_{l}^{1 s}-T_{l, 21}^{(s)} \\
& D_{l}^{N s}=T_{l, 21}^{(1)} B_{l}^{(s)}+T_{l, 22}^{(s)} .
\end{aligned}
$$

Substituting (36) into (35), the rest of the coefficients can be obtained for the DGFs.

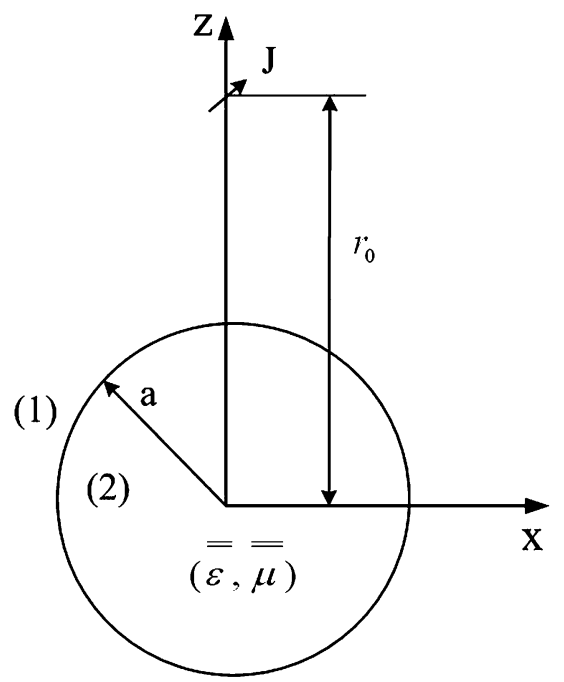

Fig. 2. Two-layer model.

3) Source in the Last Layer: When the current source is located in the last layer (i.e., $s=N$ ), the coefficients are

$$
\begin{aligned}
& {\left[\Upsilon_{l, 1 N}\right]=\left[\begin{array}{cc}
A_{l}^{1 N} & 0 \\
0 & 0
\end{array}\right]} \\
& {\left[\Upsilon_{l, f N}\right]=\left[\begin{array}{ll}
A_{l}^{m N} & 0 \\
C_{l}^{f N} & 0
\end{array}\right]} \\
& {\left[\Upsilon_{l, N N}\right]=\left[\begin{array}{ll}
0 & 0 \\
C_{l}^{N N} & 0
\end{array}\right] .}
\end{aligned}
$$

From the recurrence (27), similarly we have

$$
\left[\Upsilon_{l, f N}\right]=\left[T_{l, f-1}\right] \cdots\left[T_{l, 1}\right]\left[\Upsilon_{l, 1 N}\right]-u(f-N)\left[U_{N}\right]
$$

By letting $f=N$, the coefficient for the first region is

$$
A_{l}^{1 N}=\frac{1}{T_{l, 11}^{(1)}}
$$

For the last layer, it is found that

$$
C_{l}^{N N}=T_{l, 21}^{(1)} A_{l}^{1 N}
$$

Similarly, the rest of the coefficients can be obtained by inserting (41) into (39).

\section{Numerical VALIDATION AND ANISOTROPY SUTDY}

To further illustrate how to use the present theory in a more practical way, we study the electromagnetic radiation and scattering from a special dipole in a two-layer structure where the first and the second layer are occupied by the air and a uniaxial anisotropic sphere, respectively (see Fig. 2).

An infinitesimal electric dipole is assumed to be in the Region (1), and the center of the spherical coordinates is set to be the center of the sphere. $a$ is the radius of the sphere, and $r_{0}$ is the 
distance between the dipole and the center of the sphere. This ideal dipole is given by

$$
\begin{aligned}
\boldsymbol{J}\left(r^{\prime}\right) & =E_{m} f\left(r_{0}\right) \frac{\delta\left(r^{\prime}-r_{0}\right) \delta\left(\theta^{\prime}-\alpha\right) \delta\left(\phi^{\prime}-\beta\right)}{\left|r^{\prime}-r_{0}\right|^{2} \sin \theta^{\prime}} \hat{\boldsymbol{\theta}} \\
f\left(r_{0}\right) & =\frac{4 \pi i}{\omega \mu_{0}} r_{0} e^{i k_{0} r_{0}} .
\end{aligned}
$$

In our work, we let $\alpha=0$ and $\beta=0$, and $r_{0}$ should be infinite in order to transform a plane-wave scattering problem into a radiation problem [25]. Note that, conventionally, only plane-wave incidence is considered in the problems of scattering by anisotropic spheres. Thanks to the developed dyadic Green's function in the present paper, one can also consider current-source illumination. We consider electric scattering field in the far-zone of region (1). Hence, the scattering DGFs are constructed

$$
\begin{aligned}
& \overline{\boldsymbol{G}}_{e s}^{(11)}=\frac{i \mu_{0} k_{0}}{4 \pi} \sum_{m, n} D_{m n}\left[\boldsymbol{M}_{o m n}^{(2)}\left(k_{0}\right) B_{M}^{11} \boldsymbol{M}_{{ }_{o} m n}^{(2)}\left(k_{0}\right)\right. \\
& +\boldsymbol{N}_{o}^{(2)}\left({ }_{o} m\left(k_{0}\right) B_{N}^{11} \boldsymbol{N}_{{ }_{o} m n}^{(2)}\left(k_{0}\right)\right] \\
& \overline{\boldsymbol{G}}_{e s}^{(21)}=\frac{i \mu_{t} k_{0}}{4 \pi} \sum_{m, n} D_{m n}\left[\boldsymbol{M}_{e_{o} m v_{2}}\left(k_{t}\right) D_{M}^{21} \boldsymbol{M}_{{ }_{o} m n}^{(2)}\left(k_{0}\right)\right. \\
& \left.+\boldsymbol{N}_{e_{o} m v_{1}}\left(k_{t}\right) D_{N}^{21} \boldsymbol{N}_{{ }_{o} m n}^{(2)}\left(k_{0}\right)\right]
\end{aligned}
$$

where

$$
\begin{aligned}
& k_{0}^{2}=\omega^{2} \mu_{0} \epsilon_{0} \\
& k_{t}^{2}=\omega^{2} \mu_{0} \epsilon_{0} \mu_{t} \epsilon_{t} .
\end{aligned}
$$

The boundary conditions at $r=a$ are

$$
\begin{aligned}
\hat{\boldsymbol{r}} \times \overline{\boldsymbol{G}}_{e}^{(1)} & =\hat{\boldsymbol{r}} \times \overline{\boldsymbol{G}}_{e}^{(2)} \\
\hat{\boldsymbol{r}} \times\left[\frac{1}{\mu_{0}} \nabla \times \overline{\boldsymbol{G}}_{e}^{(1)}\right] & =\hat{\boldsymbol{r}} \times\left[\overline{\boldsymbol{\mu}}^{-1} \cdot \nabla \times \overline{\boldsymbol{G}}_{e}^{(2)}\right]
\end{aligned}
$$

where

$$
\begin{aligned}
\overline{\boldsymbol{G}}_{e}^{(2)} & =\overline{\boldsymbol{G}}_{e s}^{(21)} \\
\overline{\boldsymbol{G}}_{e}^{(1)} & =\overline{\boldsymbol{G}}_{0}+\overline{\boldsymbol{G}}_{e s}^{(11)} .
\end{aligned}
$$

where $\bar{G}_{0}$ represents the unbounded dyadic Green's function in region (1).

The scattering coefficients can thus be obtained after some manipulation

$$
\begin{aligned}
B_{M}^{11} & =\frac{\eta_{t} j_{v_{2}}\left(k_{t} a\right) \partial j_{n}\left(k_{0} a\right)-j_{n}\left(k_{0} a\right) \partial j_{v_{2}}\left(k_{t} a\right)}{h_{n}^{(2)}\left(k_{0} a\right) \partial j_{v_{2}}\left(k_{t} a\right)-\eta_{t} j_{v_{2}}\left(k_{t} a\right) \partial h_{n}^{(2)}\left(k_{0} a\right)} \\
B_{N}^{11} & =\frac{\eta_{t} j_{n}\left(k_{0} a\right) \partial h_{v_{1}}^{(2)}\left(k_{t} a\right)-j_{v_{1}}\left(k_{t} a\right) \partial j_{n}\left(k_{0} a\right)}{j_{v_{1}}\left(k_{t} a\right) \partial h_{n}^{(2)}\left(k_{0} a\right)-\eta_{t} h_{n}^{(2)}\left(k_{0} a\right) \partial h_{v_{1}}^{(2)}\left(k_{t} a\right)}
\end{aligned}
$$

$$
\begin{aligned}
D_{M}^{21} & =\frac{\eta_{t}\left[h_{n}^{(2)}\left(k_{0} a\right) j_{n}^{\prime}\left(k_{0} a\right)-h_{n}^{(2)^{\prime}}\left(k_{0} a\right) j_{n}\left(k_{0} a\right)\right]}{\mu_{t}\left[h_{n}^{(2)}\left(k_{0} a\right) \partial j_{v_{2}}\left(k_{t} a\right)-\eta_{t} j_{v_{2}}\left(k_{t} a\right) \partial h_{n}^{(2)}\left(k_{0} a\right)\right]} \\
D_{N}^{21} & =\frac{\eta_{t}\left[j_{n}\left(k_{0} a\right) h_{n}^{(2)^{\prime}}\left(k_{0} a\right)-h_{n}^{(2)}\left(k_{0} a\right) j_{n}^{\prime}\left(k_{0} a\right)\right]}{\mu_{t}\left[j_{v_{1}}\left(k_{t} a\right) \partial h_{n}^{(2)}\left(k_{0} a\right)-\eta_{t} h_{n}^{(2)}\left(k_{0} a\right) \partial h_{v_{1}}^{(2)}\left(k_{t} a\right)\right]}
\end{aligned}
$$

where $F^{\prime}(x)=(\partial F(x) / \partial x), \partial F_{v}(x)=\left(\partial\left[x F_{v}(x)\right] / x \partial x\right)$ and $F$ denotes Bessel/ $2^{n d}$-Hankel functions involved in the (47). Now, (15) can be applied to obtain electric fields. In numerical calculation, the radar cross section (RCS) is defined as [26]

$$
\operatorname{RCS}=\lim _{r \rightarrow \infty} 4 \pi r^{2} \frac{\left|E_{s}(\theta, \phi)\right|^{2}}{\left|E_{i}\left(\theta_{i}, \phi_{i}\right)\right|^{2}}
$$

where $\theta_{i}$ and $\phi_{i}$ are incident angles. The monostatic (backscattering) RCS is of our particular interest herein, and the RCS in all figures are normalized by $\pi a^{2}$ where $a$ is the radius of the sphere. The truncation number $N=40$ is chosen. It can be verified that the RCS values are convergent for bigger $\mathrm{N}$ on the workstation.

In Fig. 3, the RCS result of an isotropic sphere is compared with that of a slightly anisotropic sphere. It can be seen that the normalized RCS values are quite sensitive to the anisotropy ratio of the sphere, especially the electric size is not very small. Even a $2 \%$ difference of anisotropy ratio will result in the obvious variation of RCS. In Fig. 4, the joint anisotropy effects are discussed. Two cases are shown: 1) $\mathrm{AR}_{e}=0.9$ and $\mathrm{AR}_{m}=1.2$, and 2) $\mathrm{AR}_{e}=1.1$ and $\mathrm{AR}_{m}=1.2$. It can be found that RCS characteristics of a sphere with joint anisotropy are greatly modified by anisotropy ratio. The case of the sphere with bigger electric anisotropy ratio in Fig. 4 exhibits many zero and near-zero values of RCS, the sphere can then be considered as invisible. If the radius of the sphere or the frequency is properly chosen, invisible performance can be realized. By calculating other various cases with different anisotropy ratio, it can be verified that joint anisotropy will produce more zero RCS values than single anisotropy, and the RCS of the dielectric anisotropic sphere carries a complex form, which cannot be predicted by a simple theory.

In Figs. 5 and 6, we study the absorbing spheres with single anisotropy and joint anisotropy. In Fig. 5, it is clear that the oscillations exhibit irregular forms when $k_{0} a<10$, and for bigger values of $k_{0} a$, the oscillations start to show a regular decaying form, which agrees with the results for conducting spheres and the results for the uniaxial anisotropic spheres in [27], [28]. After considering different cases of $\epsilon_{t}$, it is also found that for absorbing anisotropic spheres, the extrema of the normalized RCS values are proportional to the imaginary part of the transverse $\epsilon_{t}$ and cannot be bigger than unit, if the rest of material's parameters keep unchanged. The RCS characteristics of absorbing spheres with joint anisotropy have been shown in Fig. 6. The RCS values of absorbing anisotropic spheres are predictable when $k_{0} a$ becomes large enough. The case of $\epsilon_{t}=$ $1-0.6 i, \mu_{t}=1-0.65 i$ in Fig. 6 is of our particular interest. It 


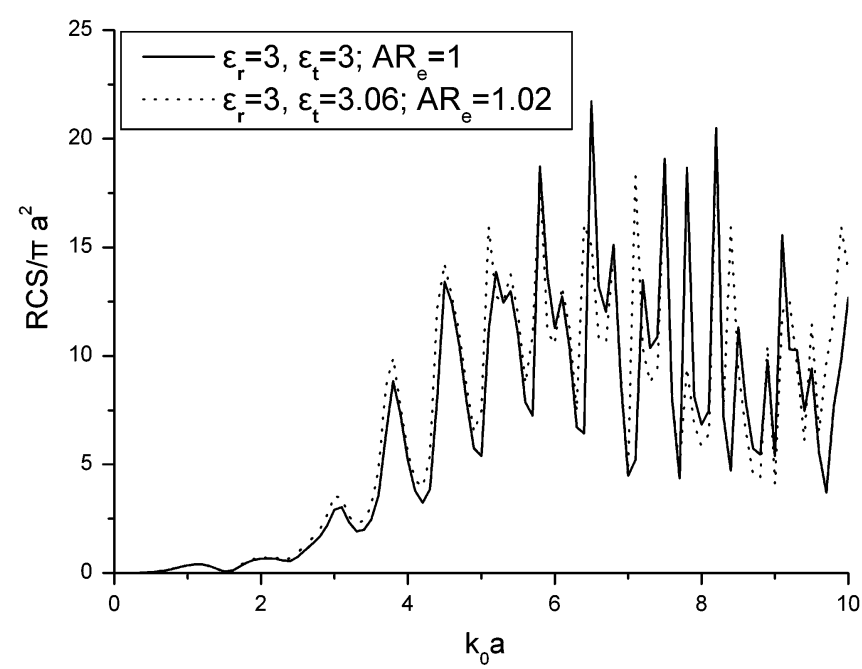

Fig. 3. Sensitivity of normalized RCS values for dielectric spheres with single anisotropy $\left(\mu_{r}=\mu_{t}=1\right)$.

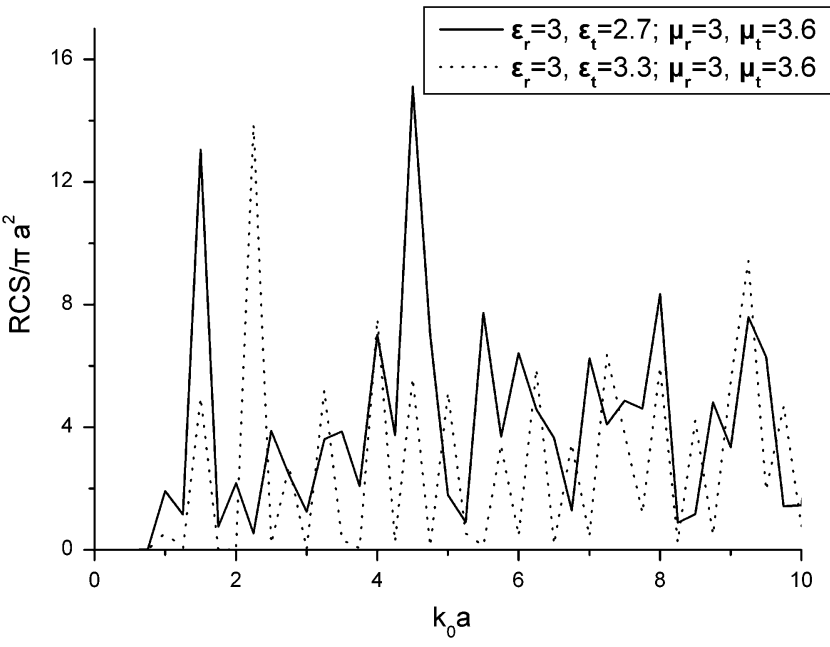

Fig. 4. Normalized RCS values versus $k_{0} a$ for dielectric spheres with joint anisotropy.

can be seen that the RCS value is very close to zero at $k_{0} a>4$. By calculating other cases whose $\epsilon_{t}$ is very close to $\mu_{t}$, similar phenomenon will be observed, which exhibits great potential in stealth technology.

It can be also observed that the values of the absorption play an important role in the scattering behavior, which make the effects of anisotropy ratio upon RCS controllable. In the lossless dielectric cases shown in Figs. 3 and 4, the scattering behavior depends on anisotropy ratio in a complex form, which is difficult to predict by a simple method. Those extrema for absorbing spheres are found to be determined by the limit $\left(\left|\left(\sqrt{\mu_{t} / \epsilon_{t}}-1 / \sqrt{\mu_{t} / \epsilon_{t}}+1\right)\right|^{2}\right)$, which also partially validates our method. The $\epsilon_{r}$ and $\mu_{r}$, which are perpendicular to the electromagnetic perturbations of the incident wave, thus have little effect on the backscattering behavior for the sufficiently large absorbing spheres. The performance of RCS of the absorbing sphere stems from the attenuation of the transmitted wave in the sphere which causes all the scattering due to the reflection at the external boundary surface [28]. The transmitted waves

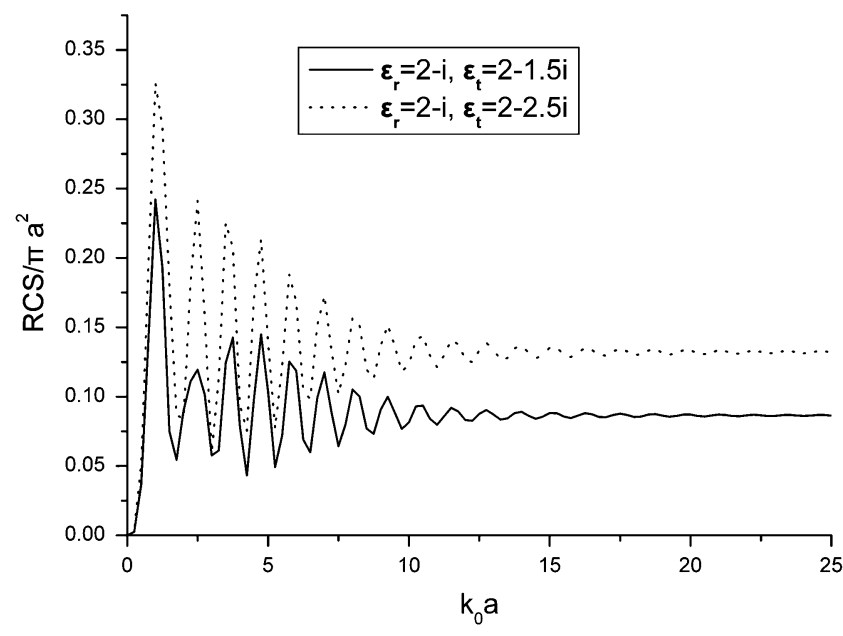

Fig. 5. Normalized RCS values versus $k_{0} a$ for absorbing spheres with single anisotropy $\left(\mu_{r}=\mu_{t}=1\right)$.

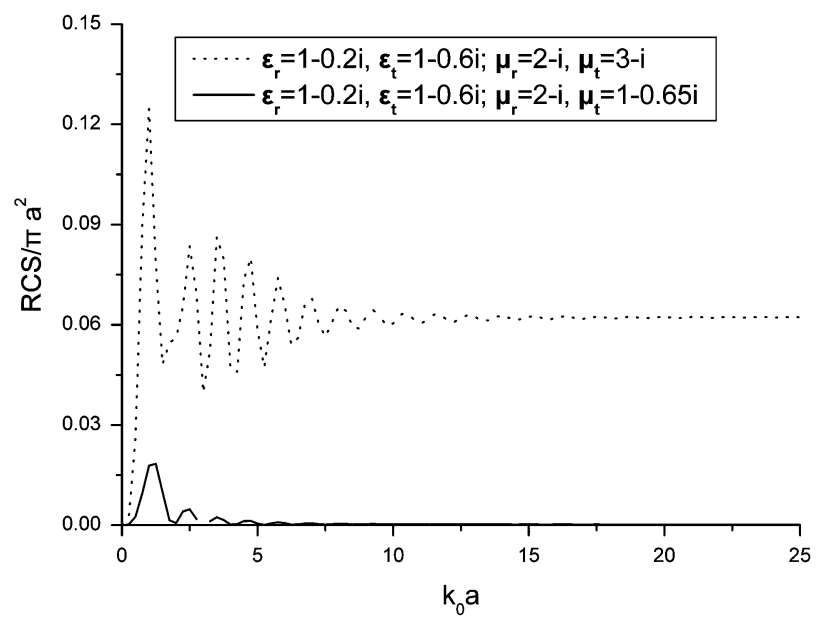

Fig. 6. Normalized RCS values versus $k_{0} a$ for absorbing spheres with joint anisotropy.

can also be computed, which is suppressed due to the length restriction. The numerical results again confirm the validity of our theoretical formulation and calculation.

\section{CONCLUSION}

This paper presents the construction of the modified spherical wave functions and the general expression of scattering DGF coefficients for multilayered uniaxial anisotropic shells. From the field expressions, the DGFs are constructed in terms of modified spherical wave functions, and the scattering DGFs can be thus obtained by using the method of scattering superposition. In the present theory, the conventional plane-wave illumination condition has been extended to arbitrary current sources, and the anisotropy effects are represented in terms of the fractional-order Bessel/Hankel functions. Based on that, the formulation of field components and Green's dyadics are greatly simplified compared to the conventional way. Since the magnetic type of DGFs can be derived by making the duality theorem, only the electric type of DGFs is analyzed herein.

Based on a recursive algorithms of the scattering coefficients which satisfy the boundary conditions of electromagnetic 
fields, the general representation of the coefficients is expressed in terms of the transmission and reflection coefficients for different cases where the current distributions are located in the first, intermediate and the last layers of the radially multilayered uniaxial anisotropic media. Then a simple geometry of radially multilayered uniaxial anisotropic media is considered and anisotropy effects are extensively analyzed.

\section{APPENDIX I}

\section{SOME PROPERTIES OF SPHERICAL BESSEL/HANKEL Functions}

In the formulation of this paper, spherical Bessel/Hankel functions are employed, which are defined as

$$
\begin{aligned}
j_{v}(x) & =\sqrt{\frac{\pi}{2 x}} J_{v+\frac{1}{2}}(x) \\
h_{v}^{(2)}(x) & =\sqrt{\frac{\pi}{2 x}} H_{v+\frac{1}{2}}^{(2)}(x)
\end{aligned}
$$

and in the calculation of RCS, the following identities have to be used for simplicity

$$
\begin{gathered}
\frac{\partial\left[x j_{v}(x)\right]}{x \partial x}=\frac{j_{v}(x)}{2 x}+\frac{1}{2}\left[j_{v-1}(x)-j_{v+1}(x)\right] \\
\frac{\partial\left[x h_{v}^{(2)}(x)\right]}{x \partial x}=\frac{h_{v}^{(2)}(x)}{2 x}+\frac{1}{2}\left[h_{v-1}^{(2)}(x)-h_{v+1}^{(2)}(x)\right] .
\end{gathered}
$$

When the argument of the second-order Hankel functions approaches a sufficiently large value, we will have the asymptotic forms

$$
\begin{aligned}
h_{n}^{(2)}(x) & \approx i^{n+1} \frac{e^{-i x}}{x}, \quad x \rightarrow \infty \\
\frac{\partial\left[x h_{n}^{(2)}(x)\right]}{x \partial x} & \approx i^{n} \frac{e^{-i x}}{x}, \quad x \rightarrow \infty .
\end{aligned}
$$

As for the associated Legendre polynomials, these properties have to be utilized in this paper

$$
\begin{gathered}
\left.\frac{\partial P_{n}^{m}(\cos \theta)}{\partial \theta}\right|_{\theta=0}=-\frac{n(n+1)}{2} \delta_{m}^{1} \\
\left.\frac{P_{n}^{m}(\cos \theta)}{\sin \theta}\right|_{\theta=0}=-\frac{n(n+1)}{2} \delta_{m}^{1}
\end{gathered}
$$

where $\delta_{m}^{1}$ denotes Kronecker delta function.

\section{REFERENCES}

[1] J. A. Kong, Electromagnetic Wave Theory, 3rd ed. New York: Wiley, 1990.

[2] C. T. Tai, Dyadic Green's Functions in Electromagnetic Theory, 2nd ed. Piscataway, NJ: IEEE Press, 1994.

[3] L. W. Li, Dyadic Green's Functions in Inhomogeneous Media. New York: Wiley, 2004.

[4] J. B. Titchener and J. R. Willis, "The reflection of electromagnetic waves from stratified anisotropic media," IEEE Trans. Antennas Propag., vol. 39, no. 1, pp. 35-39, Jan. 1991.

[5] N. K. Uzunoglu, P. G. Gottis, and J. G. Fikioris, "Excitation of electromagnetic waves in a gyroelectric cylinder," IEEE Trans. Antennas Propag., vol. 33, no. 1, pp. 90-99, Jan. 1995.
[6] G. Kobidze and B. Shanker, "Integral equation based analysis of scattering from 3-D inhomogeneous anisotropic bodies," IEEE Trans. Antennas Propag., vol. 52, no. 10, pp. 2650-2658, Oct. 2004.

[7] E. L. Tan, "Vector wave function expansions of dyadic Green's functions for bianisotropic media," Proc. Inst. Elect. Eng. Microw. Antennas Propag., vol. 149, pp. 57-63, Feb. 2002.

[8] H. T. Hui and E. K. N. Yung, "Dyadic Green's functions for the parallel-plate chirowaveguide," Proc. Inst. Elect. Eng. Microw. Antennas Propag., vol. 145, pp. 273-278, Aug. 1998.

[9] L. B. Felsen and N. Marcuvitz, Radiation and Scattering of Waves. Englewood Cliffs, NJ: Prentice Hall, 1973.

[10] E. L. Tan and S. Y. Tan, "Spectral-domain dyadic Green's functions for surface current excitation in planar stratified bianisotropic media," Proc. Inst. Elect. Eng. Microw. Antennas Propag., vol. 146, pp. 394-400, Dec. 1999.

[11] C. M. Krowne, "Green's function in the spectral domain for biaxial and uniaxial anisotropic planar dielectric structures," IEEE Trans. Antennas Propag., vol. 32, pp. 1273-1281, Dec. 1984.

[12] A. B. Gnilenko and A. B. Yakovlev, "Electric dyadic Green's functions for applications to shielded multilayered transmission line problems," Proc. Inst. Elect. Eng. Microw. Antennas Propag., vol. 146, pp. 111-118, Apr. 1999.

[13] G. W. Hanson, "Dyadic Green's function for a multilayered planar medium-a dyadic eigenfunction approach," IEEE Trans. Antennas Propag., vol. 52, no. 12, pp. 3350-3356, Dec. 2004.

[14] L. W. Li, P. S. Kooi, M. S. Leong, and T. S. Yeo, "Analytic representation of scattering dyadic Green's functions' coefficients for cylindrically multilayered chiral media," J. Electromagn. Waves Applicat., vol. 9, no. 9, pp. 1207-1221, Sep. 1995.

[15] I. V. Lindell, "TE/TM decomposition of electromagnetic scources in uniaxial anisotropic media," Microw. Opt. Technol. Lett., vol. 9, pp. 108-111, 1995.

[16] W. S. Weiglhofer, "Dyadic Green's functions for general uniaxial anisotropic media," Proc. Inst. Elect. Eng. Microw. Antennas Propag., vol. 137, pp. 5-10, 1990.

[17] I. V. Lindell and W. S. Weiglhofer, "Green dyadic for a uniaxial bianisotropic medium," IEEE Trans. Antennas Propag., vol. 42, no. 7, pp. 1013-1016, July 1994.

[18] Z. S. Wu and Y. P. Wang, "Electromagnetic scattering for mulilayered sphere: Recursive algorithms," Radio Sci., vol. 26, no. 6, pp. 1393-1401, 1991.

[19] V. I. Okhmatovski and A. C. Cangellaris, "Efficient calculation of the electromagnetic dyadic Green's function in spherical layered media," IEEE Trans. Antennas Propag., vol. 51, no. 12, pp. 3209-3220, Dec. 2003.

[20] S. M. Ali, T. M. Habashy, and J. A. Kong, "Spectral-domain dyadic Green's function in layered chiral media," J. Opt. Soc. Am. A, vol. 41, no. 3, Mar. 1993.

[21] C.-W. Qiu, L.-W. Li, T.-S. Yeo, and S. Zouhdi, "Scattering by rotationally symmetric anisotropic spheres: Potential formulation and parametric studies," Phys. Rev. E, vol. 75, no. 2, pp. 026609/1-026609/8, 2007.

[22] C.-W. Qiu, L.-W. Li, Q. Wu, and T.-S. Yeo, "Field representations in general gyrotropic media in spherical coordinates," IEEE Antennas Wireless Propag. Lett., vol. 4, no. 1, pp. 467-470, Dec. 2005.

[23] Y.-L. Geng, X.-B. Wu, L.-W. Li, and B.-R. Guan, "Electromagnetic scattering by an inhomogeneous plasma anisotropic sphere of multilayers," IEEE Trans. Antennas Propag., vol. 53, no. 12, pp. 3982-3989, Dec. 2005.

[24] J. C. Monzon, "Three-dimensional field expansion in the most general rotationally symmetric anisotropic material: Application to scattering by a sphere," IEEE Trans. Antennas Propag., vol. 37, no. 6, pp. 728-735, Jun. 1989.

[25] L. W. Li, D. You, M. S. Leong, and T. S. Yeo, "Electromagnetic scattering by multilayered chiral-media structures: a scattering-toradiation transform," Progress Electromagn. Res., vol. 26, pp. 249-291, 2000.

[26] Y. Zhang, Y. E. Yang, H. Braunisch, and J. A. Kong, "Electromagnetic wave interaction of conducting object with rough surface by hybrid SPM/MOM technique," Progress Electromagn. Res., vol. 22, pp. 315-335, 1999.

[27] K. L. Wong and H. T. Chen, "Electromagnetic scattering by a uniaxially anisotropic sphere," Proc. Inst. Elect. Eng. Antennas Propag., vol. 139, no. 4, pp. 314-318, Aug. 1992.

[28] M. Kerker, The Scattering of the Light and Other Electromagnetic Radiation. New York: Academic Press, 1969. 


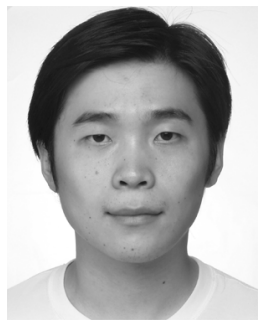

Cheng-Wei Qiu ( $\mathrm{S}^{\prime} 03-\mathrm{M}^{\prime}$ '07) was born in Zhejiang, China, on March 9, 1981. He received the B.Eng. degree from the University of Science and Technology of China, Hefei, China, in 2003 and the Ph.D. degree from the Joint Ph.D. Programme between the National University of Singapore, Singapore and the Laboratoire de Génie Electrique de Paris-Supélec (SUPELEC), France, in 2007.

His research interests are in the areas of electromagnetic wave theory, composite functional materials, and metamaterial antennas.

Dr. Qiu was the recipient of the SUMMA Graduate Fellowship in Advanced Electromagnetics for 2005. He was also the recipient of the IEEE AP-S Graduate Research Award in 2006. In 2007, he received the Young Scientist Travel Grant for ISAP2007 in Niigata, Japan, and the Travel Grant for Metamaterial'07 in Rome, Italy.

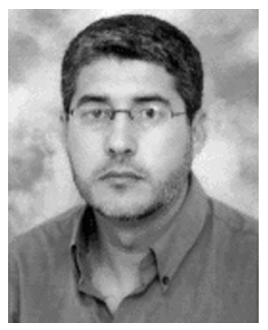

Saïd Zouhdi (SM'05) received the Ph.D. degree in electronics from the University of Pierre et Marie Curie, France, in 1994 and the Habilitation in Electrical Engineering from the University Paris Sud, France, in 2003.

From September 1995 to August 2007, he was an Associate Professor at the University Pierre et Marie Curie. In September 2007, he joined the University of Paris Sud, Orsay, France, where he is currently a Professor of electrical engineering. He is also a member of the Laboratoire de Génie Electrique de Paris, SUPELEC. He has co-edited the first book on metamaterials Advances in Electromagnetics of Complex Media and Metamaterials (Kluwer Academic, 2003) and is an Editor of the journal Metamaterials. He has published over 100 journal papers, book chapters, and conference articles. His research interests include electromagnetic wave interaction with complex structures and antennas, electromagnetics of unconventional complex materials such as metamaterials, chiral, bianisotropic and PBG materials, and optimization methods for various designs.

Dr. Zouhdi organized two NATO Advanced Research Workshops, organized and chaired various special sessions in international symposia and conferences, and has guest edited/co-edited three special issues.

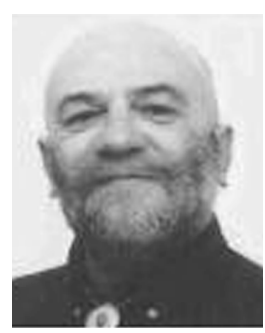

Adel Razek (F'99) was born in Cairo, Egypt. He received the Dip. Eng and M.Sc. Eng. degrees from Cairo University, in 1968 and 1971, respectively.

Since 1986, he has been a Research Director at the CNRS Centre National de la Recherche Scientifique in France. Joining the INPG Institut National Polytechnique de Grenoble in 1971, he became Docteur d'Etat ès Sciences Physiques in 1976. In 1977, he was a Postdoctoral Researcher at INPG. He moved to the Laboratoire de Génie Electrique de Paris associated to CNRS, SUPELEC and the University of Paris, as a Research Scientist at CNRS in 1978, Senior Research Scientist in 1981, Research Director in 1986, and Senior Research Director in 1997. He is the author or coauthor of over 150 scientific papers. His current primary research concerns computational electromagnetics (EMC, NDT, CAD) and design of electrical drives and actuators.

Dr. Razek is a Fellow of the Institution of Electrical Engineers (IEE) London, U.K., and a Membre Émérite of the Société des Ingénieurs Électriciens (SEE), France. He received the André Blondel medal in 1985 for his research work. 